Article:

On-demand generation and removal of alginate biocompatible microvalves for flow control in microfluidics

J. Sáez, J. Etxebarria, M. Antoñana-Díez, F. Benito-López

Sensors and Actuators B: Chemical 234:1-7 (2016)

This work is made available online in accordance with publisher policies. To see the final version of this work please visit the publisher's website. Access to the published online version may require a subscription.

Link to publisher's version:

http://dx.doi.org/10.1016/i.snb.2016.04.140

Copyright statement: (c) 2016 Elsevier Ltd. Full-text reproduced in accordance with the publisher's self-archiving policy.

This manuscript version is made available under the CC-BY-NC-ND 4.0 license http://creativecommons.org/licenses/by-nc-nd/4.0

$$
\text { (c) }
$$




\title{
ON-DEMAND GENERATION AND REMOVAL OF ALGINATE BIOCOMPATIBLE MICROVALVES FOR FLOW CONTROL IN MICROFLUIDICS
}

\author{
J. Saez ${ }^{1}$, J. Etxebarria ${ }^{2}$, M. Antonana-Diez ${ }^{2}$, F. Benito-Lopez ${ }^{1,3^{*}}$ \\ ${ }^{1}$ Microfluidics UPV/EHU Cluster, Analytical Chemistry Department, University of the \\ Basque Country UPV/EHU, Vitoria-Gazteiz, SPAIN \\ ${ }^{2}$ IK4-Ikerlan, SPAIN \\ ${ }^{3}$ Insight: Centre for Data Analytics, National Centre for Sensor Research, Dublin City \\ University, Dublin, Ireland
}

This paper describes for the first time the use of alginate hydrogels as miniaturised microvalves in microfluidic devices. These biocompatible and biodegradable microvalves are in situ generated, on demand, allowing for microfluidic flow control. The microfluidic devices were fabricated using the origami technique with a single sheet of cyclic olefin polymer folded into several layers followed by thermocompression bonding. The hydrogels can be dehydrated at mild temperatures, 37 ${ }^{\circ} \mathrm{C}$, to slightly open the microvalve and chemically erased using an ethylenediaminetetraacetic acid disodium salt dehydrated (EDTA) solution, to completely open the channel, ensuring the reusability of the whole device.

\section{INTRODUCTION}

Lab on a Chip (LOC) it is a multidisciplinary area of science that covers chemistry, physics, engineering and biotechnology, claiming the miniaturisation of devices for fluidic handling and detection. The driving force behind miniaturisation is to enhance performance gained by down-scaling analytical systems, and to integrate multiple components into a single device. ${ }^{1}$

LOC devices offer many advantages compared to other traditional analytical platforms; for instance, the reduced dimensions of microfluidic components allow for the manipulation of small volumes of fluids which lead to less reagent consumption, reduced costs and less waste generation. Temperature can be controlled and changed quickly due to the low thermal mass and large surface to volume ratio of microfluidics, which facilitates heat transfer. Moreover, the ability to couple multiple channels together, allows for easy handling of high throughput multicomponent devices and leads 
to decreased analysis times. ${ }^{2}$ Their reduced dimensions allow for the creation of portable devices for in situ testing and the potential integration of multiple components for sampling, fluid handling, detection and reporting of the results in a single run as per the Micro Total Analysis System concept.

The current state-of-the-art for microfluidic devices is based on flow systems that employ traditional pumping, valving and mixing components. These systems are generally expensive, difficult to integrate in a microfluidic device and, most of the times, can only be controlled from external sources, as for example solenoid valves ${ }^{3,4}$ Nevertheless, recent research focus has been upon improving these microfluidic components, and the resulting novel valve concepts such as "Quake" PDMS microvalves, ${ }^{5}$ "Doormat" valves ${ }^{6}$ and check valves ${ }^{7}$ among others, are now emerging. These valves have been proven to be effective and more cost effective than conventional valves but still need to be designed within the microfluidic device. The ability for ondemand in situ generation, and subsequent removal after use, of valves has not been reported yet and as such a goal is to develop novel flow systems that are cheaper and easier to fabricate based upon types of microvalves.

A very interesting concept for microvalve integration in microfluidic devices is the use of smart materials for fluid handling and control. ${ }^{8}$ In particular, hydrogels are networks of polymer chains that are highly water absorbent and that posses a substantial degree of flexibility. The ability of hydrogels to absorb water arises from hydrophilic functional groups attached to the polymeric backbone, while their resistance to be dissolved comes from the addition of crosslinkers between the polymer chains of the hydrogel. ${ }^{9}$ Due to their dynamic nature, they can be identified as stimuli-responsive materials able to undergo volumetric changes in response to physical and chemical changes in their local environment. ${ }^{10}$

In particular, calcium alginate is a water-insoluble hydrogel formed from linear copolymers of anionic polysaccharide (water soluble) and calcium cations that can chelate carboxylate groups and produce crosslinkages between polysaccharide chains. The gelling properties of alginate depend strongly upon its monomeric composition, block structure, molecular size and concentration of polymer and calcium ions. ${ }^{11}$ This polymer is one of the most used biomaterial in science due to its biocompatibility and biodegradability. ${ }^{12}$

Alginates are extremely versatile biopolymers since they have been used in a variety of technical applications as biomedical ${ }^{13}$ and pharmaceutical ${ }^{14}$ and extensively used in 
the food industry because it is a powerful thickening, stabilising, and gel-forming agent. Incomprehensibly, in microfluidic applications, alginates are rarely used, primordially for reagent storage in chips and for the fabrication of microcapsules in drug delivery systems. $^{15}$

Recent advances in microtechnology for biomedical applications, resulting in products appearing on the market, ${ }^{16}$ have increased the necessity to integrate stimuli responsive materials with biocompatible capabilities within microfluidic devices making gels obtained from natural polymers are good candidates. ${ }^{17}$ In particular the use of smart materials as actuators, with innocuous chemical characteristics, will create opportunities for the next generation of novel microfluidic devices for biological applications.

This study demonstrates, for the first time, the use of calcium alginate hydrogels as miniaturised valves in microfluidic devices, as innovative alternatives to conventional hydrogel microvalves. These biocompatible and biodegradable microvalves can be generated on demand and in situ to introduce microfluidic flow control. Since calcium alginate is dehydratated at room temperature (syneresis process), it can be thermally actuated at mild temperatures, slightly unblocking the channel and, in turn, restoring the flow rate, allowing to chemically erase the microvalve from the main channel simply using an ethylenediaminetetraacetic acid disodium salt dehydrated (EDTA) solution, and so ensuring the reusability of the whole device.

\section{EXPERIMENTAL}

\subsection{Materials}

Sodium alginate was purchased by Sigma-Aldrich (St Louis, MO, USA). Calcium chloride dehydratated (Sigma-Aldrich) was used to prepare-calcium alginate hydrogel. Ethylenediaminetetraacetic acid disodium salt (EDTA) was purchased by Merck (Darmstadt, Germany) and was used for chemically erase the calcium alginate hydrogel. All the solutions were prepared using deonised (DI) water from a Milli-Q water purification system (Millipore, Milford, MA).

Isopropyl alcohol (IPA) was purchased by Panreac Química S.L.U. (Spain). Rolls of $100 \mu \mathrm{m}$ thick of COP films (ZeonorFilm®) were obtained from Zeon Chemicals (Düsseldorf, Germany). This material was chosen because of its good properties such as biocompatibility, acids and basis attack resistant and transparency.

Accura Amethyst was purchased from (3D Systems) for the fabrication of the luers 
by stereolitography technique (Viper SLA systems, 3DSystems) and were coupled to the device with screws.

The microfluidic device flows were controlled using a WPI SP120PZ syringe pump (Shangai, China). Alternatively, solutions were injected with a Harvard Apparatus model 11 elite syringe pump (Holliston, MA, USA). A MFCS ${ }^{\mathrm{TM}}-\mathrm{EZ}$ pressure driven flow controller (Fluigent, Paris, France) was used for the controlled injection of solutions. A SLG-0075 flow sensor (Sensirion CMOSens ${ }^{\circledR}$, Switzerland) was connected for the monitoring of solutions inside the microfluidic device.

\subsection{Chip Fabrication}

Microfluidic devices were designed and fabricated by the Origami technique ${ }^{18}$ by rapid prototyping using the FC-8000-60 cutting plotter from Graphtec (Irvine, CA). The 3D design was sliced into several 2D layers, which were cut with the cutting plotter, assembled and then bonded by thermocompression. ${ }^{19}$

Stereolithography 3D-printed interconnections for fluidic handling were in house fabricated and coupled to the device with screws [www.microliquid.com]. These interconnections are coupled to syringes type $\mathrm{ICO}+3,1 \mathrm{~mL}$ (Novico Medica, Barcelona, Spain) and/or to male PMMA luers (Chipshop, Jena, Germany) and Tygon 0.8 x 2.4 mm tubes (Colmer Palmer,Vernon Hills, USA).

\subsection{Hydrogel preparation}

For the hydrogel preparation, water solutions of $4 \%$ of calcium chloride and $1 \%$ of sodium alginate were prepared. ${ }^{20}$ When the sodium alginate is added to a calcium chloride solution, the calcium ions replace the sodium ions in the polymer and form the gel. The "egg-box model" for the formation of alginate polymers in the presence of alkaline metals was first described by Rees et al., ${ }^{21}$ see Figure 1.The model describes that divalent cations, such as $\mathrm{Ca}^{2+}$, are coordinated with in the cavities of alginate chains. ${ }^{22}$ A second alginate strand can also connect at the calcium ion, forming a link in which the calcium ion attaches two alginate strands together. The result is a chain of calcium crosslinked alginate strands that form a solid polymer. 


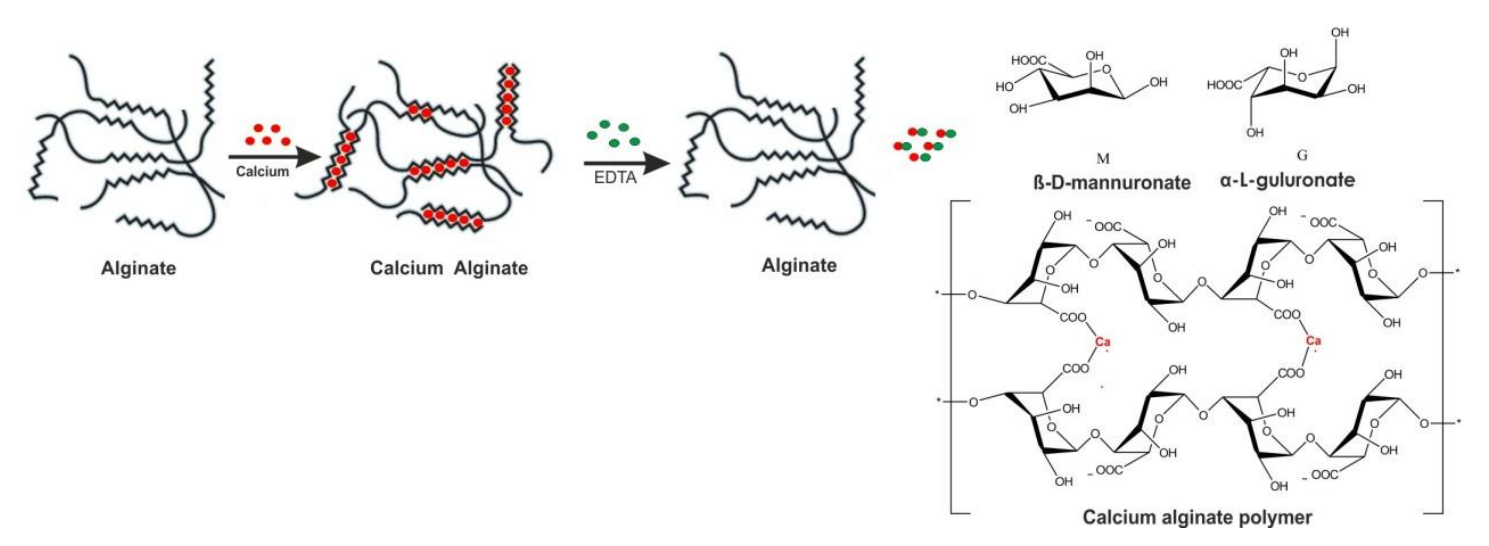

Figure 1: Schematic of the calcium alginate polymer formation and EDTA erase (left) and chemical structure of the calcium alginate formation (right).

In order to perform this reaction within the microfluidic device and generate a functional microvalve, in a controlled manner, calcium alginate beads were first synthesised on bench. For this, a syringe was filled with the sodium alginate solution and few drops of the liquid $(500 \mu \mathrm{L})$ were added to a calcium chloride solution bath, allowing polymerisation. The reaction was then stopped by putting the beads into a water bath.

\section{RESULTS AND DISCUSSION}

\subsection{Chip Fabrication}

In order to first prove the concept of valve generation, the first generation prototype microfluidic device (Prototype 1) was designed with a simple T-shape configuration containing seven $100 \mu \mathrm{m}$ thick COP layers, with a main channel stream of $1 \mathrm{~mm}$ width and a perpendicular channel of $500 \mu \mathrm{m}$ for microvalve generation. A more sophisticated second generation microfluidic design (prototype 2) was later fabricated in order to independently control the injection of the $\mathrm{CaCl}_{2}$, EDTA and alginate solutions into the main channel stream. This second generation was fabricated including an additional set of layers of COP, where the EDTA microchannel is placed with a V-shape $(1 \mathrm{~mm}$ width, $200 \mu \mathrm{m}$ height) above the generated microvalve in order to independently control the gel microvalve removal. COP was used in order to guarantee the biocompatibility of the whole device

Figure 2 (A) shows an unfolded Origami prototype 1 sliced in seven layers of COP and $(\mathrm{C})$ prototype 2 sliced in nine layers of COP. The microfluidic devices contain the 
inlets/outlet, the main channel and the perpendicular channel in prototype 1 and an additional $\mathrm{V}$-shape channel in prototype 2. Figure 2 (B and D) presents the prototype 1 and prototype 2 respectively, after thermocompression with the stereolithography interconnections coupled with screws. This type of interconnector guarantees no leakage during the experiment up to 1 bar of pressure and has the possibility of being connected directly to syringe or to commercially available fittings coupled to a tubing and then to the syringe.

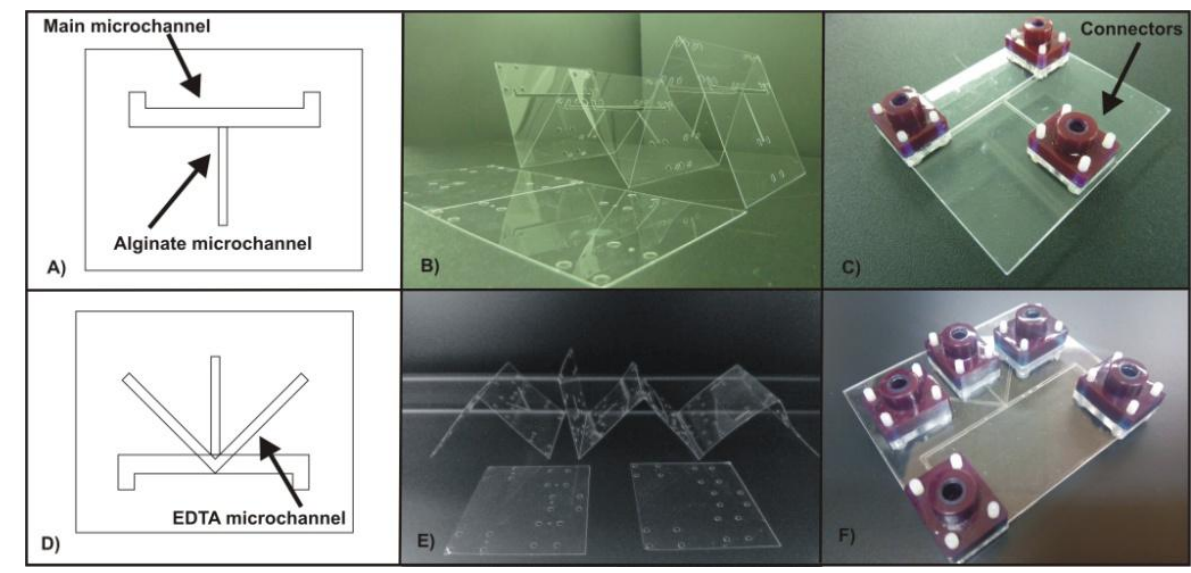

Figure 2: Prototype 1 schematic design of the microfluidic device (A), unfolded origami sheets $(B)$ and completed device following thermocompression of the origami layers with inlets/oultets $(C)$. Prototype 2 schematic design of the microfluidic device including additional V-shaped EDTA channel (D), unfolded origami sheets (E) and completed device after thermocompression of the origami layers with inlets/oultets $(F)$.

\subsection{Hydrogel characterisation}

Two approaches were followed to characterise the optimised conditions for calcium alginate microvalve generation and removal in the microfluidic device. Firstly, the percent weight/volume $(\% \mathrm{w} / \mathrm{v})$ for each sodium alginate/water and calcium chloride solutions was optimised on bench. Secondly, the flow rate of each solution into their corresponding channels was investigated.

For the characterisation of the optimum $\% \mathrm{w} / \mathrm{v}$ per solution, several proportions of sodium alginate were tested. $0.6 \%, 1 \%, 2 \%$ and $4 \% \mathrm{w} / \mathrm{v}$ of sodium alginate solution were tested in order to select the most adequate solution to be employed in the microfluidic device. It was found that $1 \% \mathrm{w} / \mathrm{v}$ sodium alginate ensures rapid, homogeneous polymerisation and low viscosity to facilitate the flow of alginate solution 
into the microfluidic device. The $\mathrm{CaCl}_{2}$ concentration was optimised by selecting the concentration of $\mathrm{CaCl}_{2}$ which shows the best performance with respect to the structural resistance of the formed calcium alginate polymer $(\eta)$. This result was obtained for $4 \%$ w/v calcium chloride solution, obtaining the same results as the work previously described by Blandino et al. ${ }^{11}$ Using this percentage, the structural resistance (robustness) of calcium alginate polymers is uniform. It was observed that $\eta$ tends to decrease when more concentrated solutions were used. This reduction in $n$ value is known to be due to the increase in diffusional resistance that $\mathrm{Ca}^{2+}$ ions suffer in their flux through a thicker membrane ( $n$, decreases with increasing diffusional resistance) and similar behaviour was reported by Yamagiwa et al. ${ }^{15}$

In order to chemically erase the microvalve an aqueous solution of $0.5 \mathrm{M}$ water EDTA was found to successfully dissolve the polymer.

\subsection{Alginate microvalve in situ generation}

The prototype 1 device was connected to the pressure driven flow controller that controls the pressure and flow of calcium chloride solution independently from sodium alginate solution into the microfluidic device. As highlighted in the experimental section the microvalves were in situ fabricated using $1 \% \mathrm{w} / \mathrm{v}$ sodium alginate and $4 \%$ w/v $\mathrm{CaCl}_{2}$ solution.

For the fabrication of the microvalve, the $\mathrm{CaCl}_{2}$ solution was flowed (Figure 3-1) through the main microchannel using the pressure driven flow controlled at $60 \mu \mathrm{L} \mathrm{min}{ }^{-1}$ through the $1 \mathrm{~mm}$ diameter channel while the alginate solution coming from the perpendicular channel reaches the T-intersection section of the microchannel $(\sim 1500$ $\mu \mathrm{m}$ length, Volume: $1.05 \mu \mathrm{L})$ after a small air plug ( $200 \mu \mathrm{m}$ length) as a manually controlled fast flow pulse using a syringe, Figure 3-2 and 3-3. Figure 3-3 presents an in situ generated microvalve in the main microchannel and in the top right figure an alginate bead fabricated outside the microfluidic device for comparison. It is appreciated in Figure 3-3 that both generated alginate polymers (on-chip and bench) present similar physical configurations with no appreciable fabrication damages or cracks. In the intersection, the calcium cations of the $\mathrm{CaCl}_{2}$ solution penetrate by diffusion into the sodium alginate solution plug and start polymerising the alginate, resulting in hydrogel formation. By introducing the alginate into the calcium solution, anisotropic polymerised particles with broad size distributions are formed at first due to differences 
in the diffusion rate of calcium throughout the alginate. ${ }^{23}$ The amount of sodium alginate solution and the shape of the generated microvalve in the microfluidic device are controlled by the speed of the alginate plug. It should be noted here that it is quite difficult to control the shape of the microvalve since the hydropobicity of the COP microchannel is not enough to prevent the alginate solution expanding throughout the main microchannel, see the shape of the microvalve in Figure 3-3. Nevertheless, the generated alginate plug is sufficient to ensure a physical barrier to close the main microchannel and act as a microvalve. A well defined microvalve could be generated by mechanically controlling the sodium alginate solution plug.

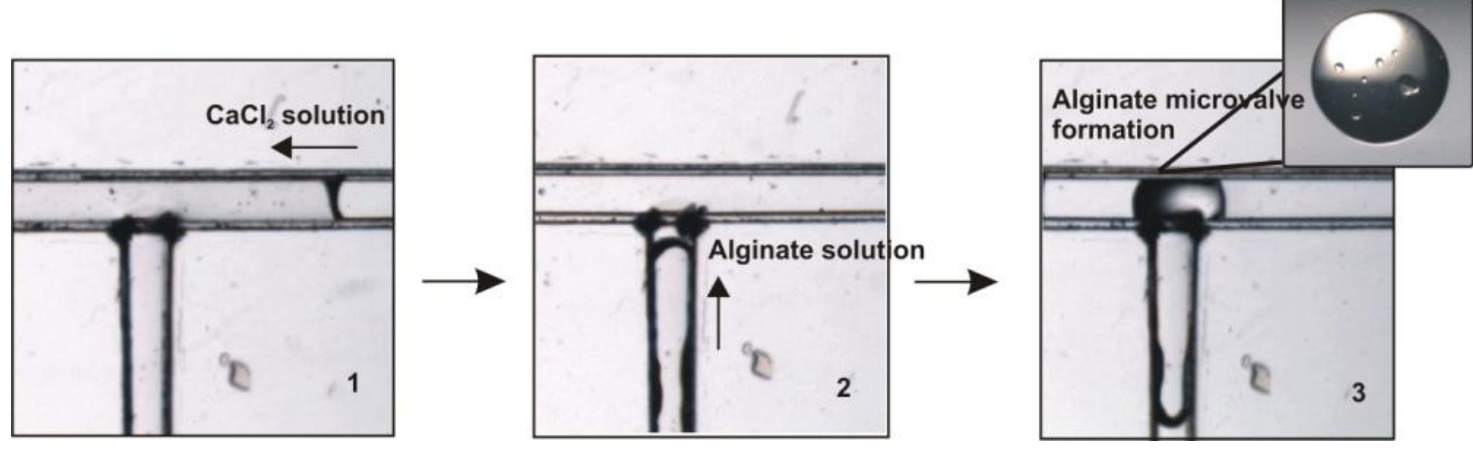

Figure 3: Time lapse images illustrating alginate microvalve formation: (1) $\mathrm{CaCl}_{2}$ solution injected into main channel, (2) Alginate solution added through feed channel and (3) Calcium alginate bead formation upon reaction of two solutions. (3) inset is a calcium alginate bead generated outside of microfluidic channel for comparison to insitu bead produced for valving applications.

\subsection{Alginate microvalve characterisation}

Figure 4 (a) shows the flow profile within the microfluidic channel during in situ microvalve formation. The flow sensor was placed after the microfluidic device and the $\mathrm{CaCl}_{2}$ solution flowed through the main channel (ON configuration) at $3 \mu \mathrm{L} \mathrm{min}^{-1}$ in order to be able to observe the formation of the microvalve. The flow in the main channel decreased to almost $0 \mu \mathrm{L} \min ^{-1}$ when the microvalve is fully formed and the channel is blocked (OFF configuration). The microvalve formation time was $40 \pm 7 \mathrm{~s}, \mathrm{n}$ $=4$ for this particular microfluidic configuration.

Although the experimental conditions of both the on-bench and in situ microvalves formation are not entirely equivalent, the formation time of the in situ microvalves correlates well with the formation of a calcium alginate beads on bench. On bench, the 
calcium alginate starts polymerising instantly at the surface of the sodium alginate bead, upon addition of $\mathrm{CaCl}_{2}$, maintaining the shape of the bead. As time progresses, the alginate drop becomes firmer with time while in contact with the $\mathrm{CaCl}_{2}$ solution. The core of the sodium alginate bead/microvalve is initially "unreactive" but over time the ion exchange moves towards the centre from the surface and forms a complete calcium alginate structure, as described by $\mathrm{Kim}^{24}$

The fluctuations in the flow rate, with a maximum of approximately $0.20 \mu \mathrm{L} \mathrm{min}^{-1}$, could be attributed to fluctuations of the flow due to reorganisation of the alginate polymer during formation or accommodation of the generated polymer to the channel configurations. The reason why the flow signal does not reach $0 \mu \mathrm{L} \min ^{-1}$ may be attributed to:

(1) the alginate polymer porosity; this can be reduced by increasing the immersion time of alginate in the calcium solution and/or decreasing the ratio between mannuronic units and guannuronic units $(\mathrm{M} / \mathrm{G})$ in the sodium alginate. Calcium alginate films with higher concentration of $\mathrm{G}$ were proven to have significantly lower water vapour permeabilities (WVP) compared to films with higher concentration of M. It was reported that the immersion time and the $\mathrm{M} / \mathrm{G}$ ratio of sodium alginate are therefore the key steps for producing calcium alginate films with low WVP and so less porosity. ${ }^{25}$

(2) inadequate assembly of the alginate microvalve with the microchannel walls. In order to minimise this effect, other T-junction configurations with a more circular or oval shape could be fabricated to better accommodate the generated microvalve.

In order to characterise the pressure resistance of the microvalve, microvalve failure pressure experiments were carried out. The flow sensor was located before the prototype 1 device and a $60 \mu \mathrm{L} \mathrm{min}^{-1}$ flow of $\mathrm{CaCl}_{2}$ was set into the main microchannel meanwhile a controlled pulse flow of sodium alginate was applied into the perpendicular microchannel, see experimental. At the T-junction the polymerisation of the calcium alginate took place resulting in the formation of the microvalve. Once the $\mathrm{CaCl}_{2}$ flow decreased to virtually $0 \mu \mathrm{L} \min ^{-1}$ the microchannel is its blocked (OFF) configuration. In this particular experiment the microvalve formation appears negative due to the large fluctuation generated in the flow sensor signal when the alginate solution is injected into the main channel. Failure pressure experiments were then carried out then by applying incremental pressures to the microvalve, through the main channel, when the microvalve is OFF. The failure pressure value of the microvalve was 
tested for $0.02,0.05,0.1$, and 1 bar and it was observed that some of the microvalves could hold up to 1 bar of pressure. However the observed fluctuations in the flow signal after applying 1 bar of pressure imply that the microvalve is not stable at this pressure for an extended period of time.

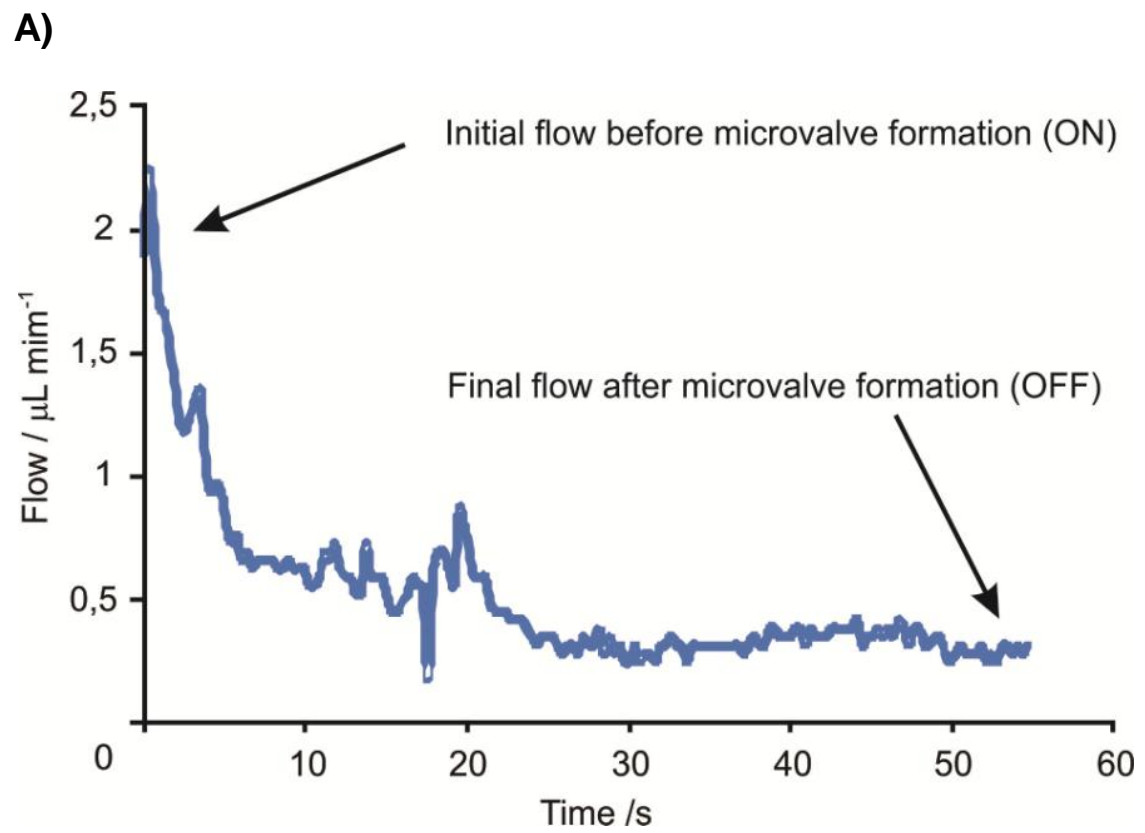

B)

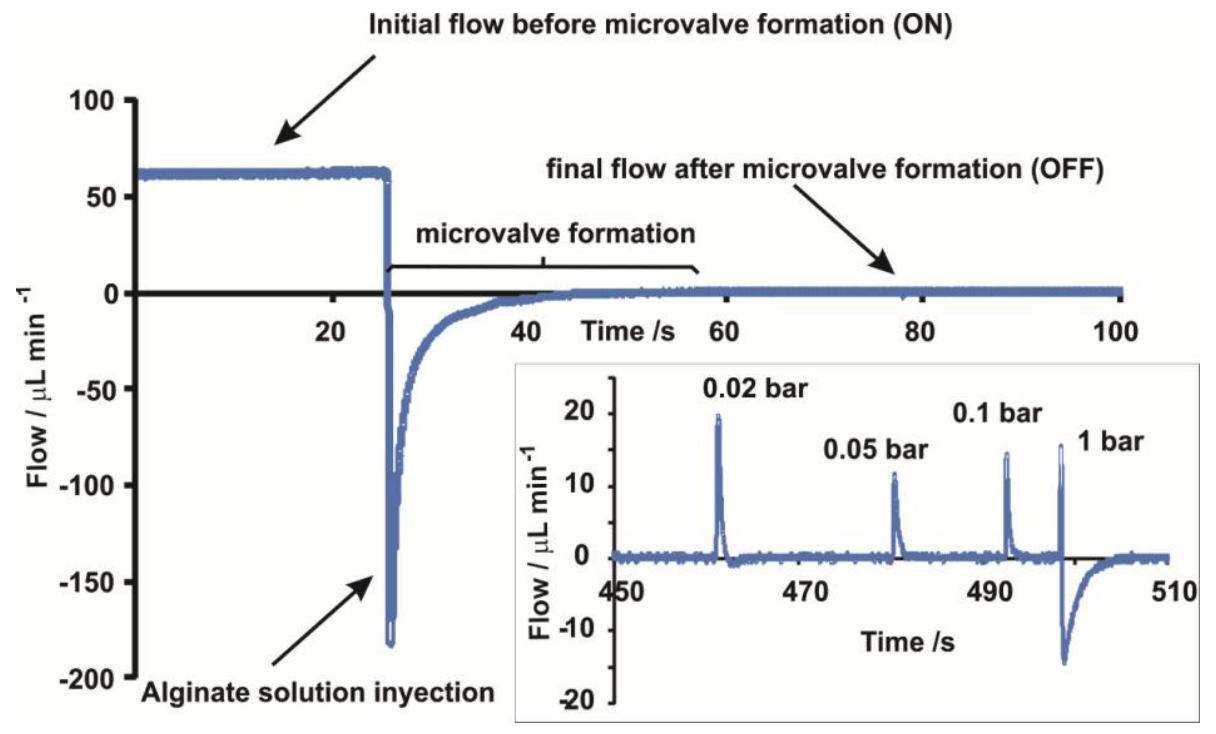

Figure 4: (a) Flow profile of prototype 1 device in the main channel of the microfluidic device during alginate microvalve fabrication (flow sensor placed at the outlet of the microfluidic device). (b) Flow profile in the main channel during alginate microvalve fabrication (flow sensor placed at the inlet of the microfluidic device). Inset: microvalve pressure failure experiments, carried out with the same microvalve. 


\subsection{Alginate microvalve actuation}

As calcium alginate exhibits the phenomenon of syneresis the polymer can therefore be actuated by temperature. Syneresis is the spontaneous release of bound water with contraction of the hydrogel volume. Therefore the blocking action generated by the microvalve in the microchannel can be partially reverted at a mild temperatures, $37^{\circ} \mathrm{C}$, as illustrated in Figure 5 with demonstrator beads on bench and in situ within a microfluidic device. Although this actuation is not reversible, the microvalve is able to shrink up to $20 \%$ of its initial volume, allowing for the liquid to flow again.

This syneresis effect of the microvalve facilitates noncontact operation and therefore independent manipulation of liquids flow in multiple microfluidic channels within an integrated microfluidic manifold simply by positioning heaters below the microvalves. It is clear that such dehydration of the hydrogel microvalves has the potential to greatly improve fabrication processes. Moreover, this thermal process could be used to allow for the injection, through the main microchannel, of an EDTA solution to completely erase the alginate microvalve when the microvalve completely blocks the microchannel when using very simple microfluidic configurations such as that represented by protototype 1 .

\section{Bench}

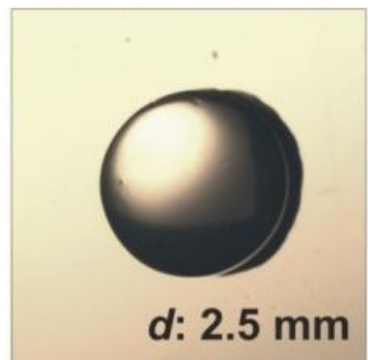

On chip

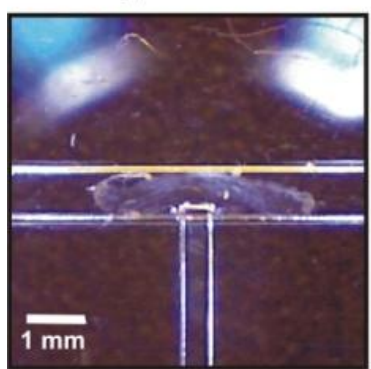

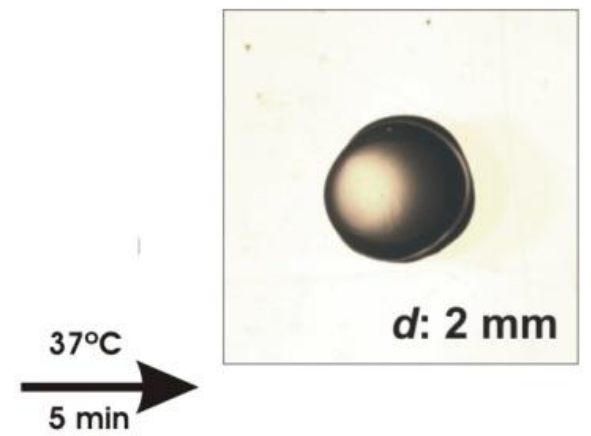

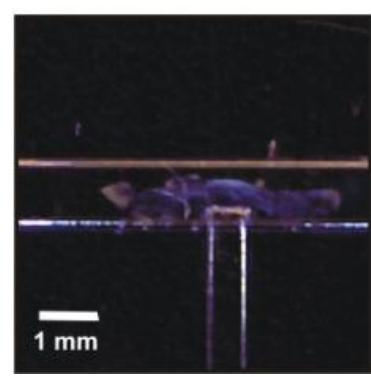


Figure 5: Dehydration of an alginate bead on bench and chip, respectively.

\subsection{Removal of the alginate microvalve}

The stability of calcium alginate hydrogel depends on the stability of calcium complexes within the hydrogel. ${ }^{26}$ Thus, chelating agents that strongly bind calcium such as sodium citrate and EDTA quickly solubilise the polymer.

Figure 6 presents the flow profile in the main microchannel when a microvalve was generated and subsequently erased with EDTA. $\mathrm{A} \mathrm{CaCl}_{2}$ solution was inserted into the prototype 2 at $500 \mathrm{~nL} \min ^{-1}$ meanwhile the alginate was inserted as a fast pulse perpendicularly. At the T-junction polymerisation of the microvalve takes place, blocking the channel and producing a decrease in the flow to virtually $0 \mu \mathrm{L} \mathrm{min}{ }^{-1}$ (Figure 6, picture 1). Following this, the addition of the EDTA chelate complex (500 nL $\left.\min ^{-1}\right)$, coming from the channel set on top of the main channel ( $\mathrm{V}$-shape) removes the calcium ions from the alginate polymer and erases the microvalve from the main microchannel increasing then the flow rate (Figure 7, picture 2). It is important to note here that for this microfluidic configuration (prototype 2) it is not necessary to heat the microvalve, since the EDTA channel sits on top of the main channel. Both channels are connected through a small orifice that gets blocked when microvalve forms.

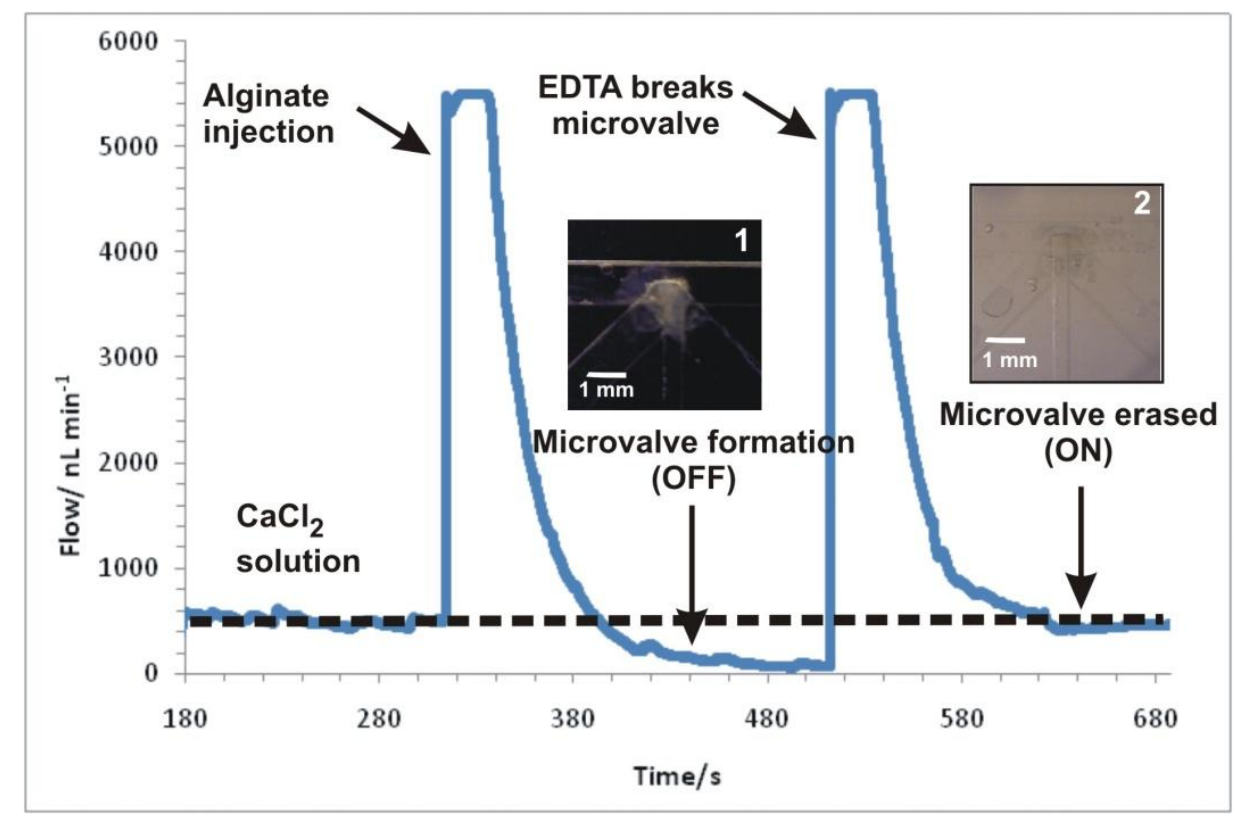

Figure 6: Flow profile in the prototype 2 main microchannel during alginate microvalve generation and removal by EDTA (flow sensor placed at the outlet of the microfluidic 


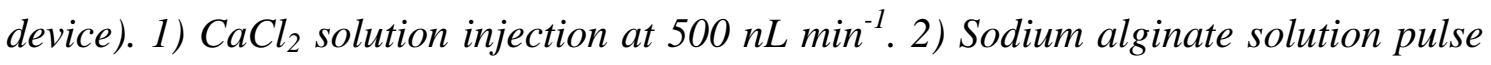
injection. 3) Generated calcium alginate microvalve (OFF) (picture 1). 4) EDTA solution injection and removal of the microvalve from the main microchannel (ON) (picture 2).

It was found out that $60 \pm 5 \mu \mathrm{L}(\mathrm{n}=3)$ of EDTA solution it is sufficient to completely erase the calcium alginate microvalve from the channel thus restoring the main microfluidic microchannel for subsequent operations. The time needed to remove the valve was approx. $120 \mathrm{~s}$ (depending on the size of the generated microvalve). The result is a fast process that is comparable to the actuation mechanism observed by others when using conventional hydrogel microvalves in microfluidic devices (e.g. photoswitchables $(60 \mathrm{~s})^{27}$ or thermoresponsive $(120 \mathrm{~s})^{18}$ to open).

In the near future, a way of controlling the dimensions of the calcium alginate microvalves will be investigated. It is believed that this preliminary investigation opens up the use of calcium alginate materials as microvalves for many applications, such as POC devices that require biocompatible and biodegradable components.

\section{CONCLUSIONS}

This contribution demonstrates the potential for alginate polymers to be used as building block materials for the fabrication of in situ microvalves within microfluidic devices with an ON/OFF actuation capability. Alginate has proven to efficiently act as actionable microvalve at mild temperatures allowing for flow control due to its syneresis effect. Moreover, by carefully adjusting the microfluidic channel configuration alginate microvalves can be easily erased with EDTA solution, ensuring the reusability of the whole device.

Alginate is biodegradable, therefore is an attractive material for microfluidic applications related to the health and food industry. Moreover these calcium alginate microvalves are low cost to produce in terms of materials, and the in situ fabrication opens the possibility of creating large arrays of microvalves in complex microfluidic structures.

\section{ACKNOWLEDGEMENTS}

Fernando Benito-Lopez acknowledges the Ramón y Cajal Programme (Ministerio de Economía y Competitividad), Spain. This project has received funding from the European Union's Seventh Framework Programme (FP7) for Research, Technological 
Development and Demonstration under grant agreement no. 604241. Authors are grateful to IK4-IKERLAN for letting them to use its facilities for the fabrication of the microfluidic devices and to Prof. Marian Martinez de Pancorbo for let us use her laboratory facilities at UPV/EHU.

\section{REFERENCES}

1 S. Haeberle and R. Zengerle, Lab Chip, 2007, 7, 1094-1110.

2 C. A. Baker, C. T. Duong, A. Grimley and M. G. Roper, Bioanalysis, 2009, 1, 967-975.

3 A. Fonseca, I. M. Raimundo Jr, J. J. Rohwedder, R. S. Lima and M. C. Araujo, Anal. Bioanal Chem., 2010, 396, 715-723.

4 A. Milani, P. J. Statham, M. C. Mowlem and D. P. Connelly, Talanta, 2015, 136, 15-22.

5 G. Pasirayi, S. M. Scott, M. Islam, L. O'Hare, S. Bateson and Z. Ali, Talanta, 2014, 129, 491-498.

6 M. Rothbauer, D. Wartmann, V. Charwat and P. Ertl, Biotechnol. Adv.,2015, 33, 948961 .

7 W. Al-Faqheri, F. Ibrahim, T. H. G. Thio, M. M. Aeinehvand, H. Arof and M. Madou, Sens. Actuators, A, 2015, 222, 245-254.

8 http://www.intechopen.com/books/advances-in-microfluidics/smart-microfluidicsthe-role-of-stimuli-responsivepolymers-in-microfluidic-devices.

9 E. M. Ahmed, J. Adv. Res., 2015, 6, 105-121.

10 D. T. Eddington and D. J. Beebe, Adv. Drug Deliv. Rev., 2004, 56, 199-210.

11 A. Blandino, M. Macías and D. Cantero, J. Biosci. Bioeng., 1999, 88, 686-689.

12 J. Sun and H. Tan, Materials, 2013, 6, 1285-1309.

13 K. Y. Lee and D. J. Mooney, Prog. Polym. Sci., 2012, 37, 106-126.

14 A. Dalmoro, A. A. Barba, G. Lamberti, M. Grassi and M. d'Amore, Adv. Polym. Technol., 2012, 31, 219-230.

15 Y. Yajima, M. Yamada, E. Yamada, M. Iwase and M. Seki, Biomicrofluidics, 2014, 8, 024115-024115-11.

16 E. Ghafar-Zadeh, Sensors (Basel), 2015, 15, 3236-3261. 
17 V. Zamora-Mora, D. Velasco, R. Hernández, C. Mijangos and E. Kumacheva, Carbohydr. Polym., 2014, 111, 348-355.

18 F. Benito-Lopez, M. Antonana-Diez, V. F. Curto, D. Diamond and V. Castro-Lopez, Lab Chip,2014, 14, 3530-3538.

19 C. L. Cassano, A. J. Simon, W. Liu, C. Fredrickson and Z. H. Fan, Lab Chip, 2015, 15, 62-66.

20 S. K. Bajpai and S. Sharma, React Funct Polym, 2004, 59, 129-140.

21 C. W. McCleary, D. A. Rees, J. W. B. Samuel and I. W. Steele, Carbohydr. Res., 1967, 5, 492-495.

22 L. Li, Y. Fang, R. Vreeker, I. Appelqvist and E. Mendes, Biomacromolecules, 2007, 8, 464-468.

23 I. Fernández Farrés and I. T. Norton, Food Hydrocoll., 2014, 40, 76-84.

24 H. Kim, Korean J. Chem. Eng., 1990, 7, 1-6.

25 G. I. Olivas and G. V. Barbosa-Cánovas, LWT - Food Sci. and Technol., 2008, 41, 359366.

26 M. Kobaslija and D. T. McQuade, Biomacromolecules, 2006, 7, 2357-2361.

27 J. ter Schiphorst, S. Coleman, J.E. Stumpel, A. Ben Azouz, D. Diamond, A.P.H.J. Schenning, Chem. Mater., 2015, 27 , 5925-5931. 